\title{
Tractional Retinal Detachment
}

National Cancer Institute

\section{Source}

National Cancer Institute. Tractional Retinal Detachment. NCI Thesaurus. Code 6118759.

Retinal detachment secondary to vasoproliferative changes in the retina and/or vitreous. 